Artigo / Article

\title{
Prevalência e caracterização da anemia em idosos do Programa de Saúde da Família
}

\author{
Prevalence and characteristics of anemia in an elderly population attending a \\ Health Family Program
}

Diana L. Barbosa ${ }^{1}$

Ilma K. G. Arruda

Alcides S. Diniz ${ }^{3}$

\begin{abstract}
A anemia é considerada um problema de saúde pública em escala mundial e é o distúrbio hematológico de maior prevalência que acomete a população idosa. O objetivo deste trabalho foi estimar a prevalência e características da anemia em idosos do Programa de Saúde da Família de Camaragibe, PE. O delineamento foi transversal, com amostra aleatória sistemática de 284 idosos $\geq 60$ anos, de ambos os sexos, realizado em novembro/dezembro-2003. A anemia foi avaliada pela hemoglobina $(\mathrm{Hb})$, concentração de hemoglobina corpuscular média (CHCM), volume corpuscular médio (VCM) e amplitude de distribuição eritrocitária $(R D W)$. A prevalência de anemia foi, em média, de 11,0\%. A morfologia eritrocitária não mostrou associação com as concentrações de Hb. A grande maioria dos idosos apresentou anemia normocrômica, normocítica, sem anisocitose, sugestivo de anemia por doença crônica. A ínfima prevalência de microcitose e macrocitose com anisocitoseminimiza a gênese da deficiência de ferro, bem como da deficiência de vitamina $B_{12}$ e ácido fólico na etiologia da anemia em idosos. Concluímos que o uso de indicadores que reflitam o grau de anisocitose eritrocitária associados àqueles que avaliam o estado nutricional do ferro é extremamente recomendado para o diagnóstico das anemias em idosos. Rev. bras. hematol. hemoter. 2006;28(4):288-292.
\end{abstract}

Palavras-chave: Anemia; idosos; índices hematimétricos; diagnóstico; Programa de Saúde da Família.

\section{Introdução}

A anemia tem sido definida como a redução patológica da concentração de hemoglobina (Hb) circulante, desencadeada por mecanismos fisiopatológicos diversos. ${ }^{1} \mathrm{~A}$ anemia é o problema hematológico mais comumente encontrado nos indivíduos idosos. Beghé et $a l,{ }^{2}$ em sua revisão sobre o tema, encontraram uma grande variação da prevalência de anemia, entre os estudos realizados com a população idosa, com cifras oscilando entre 2,9\% a 61,0\%, em homens, e 3,3\% a 41,0\%, em mulheres.
Os tipos de anemia mais prevalentes nesse grupo populacional são a anemia por doença crônica (ADC) e anemia por deficiência de ferro (ADF). ${ }^{3}$ Pesquisas recentes, envolvendo idosos americanos, demonstraram que as prevalências de ADF têm sido superadas pelas prevalências da ADC, neste grupo populacional. ${ }^{3,4}$ A diferenciação entre anemia de doenças crônicas e deficiência de ferro deve ser cuidadosamente investigada em pacientes idosos. O diagnóstico diferencial da anemia, com o objetivo de caracterizar o tipo de anemia na população idosa, poderá ser realizado com exames clínicos e laboratoriais que incluam os indicadores

${ }^{1}$ Nutricionista. Mestre em Nutrição - UFPE.

${ }^{2}$ Nutricionista. Doutor em Nutrição - UFPE. Professor adjunto do Departamento de Nutrição - UFPE.

${ }^{3}$ Médico. Doutor em Nutrição - UFPE. Professor adjunto do Departamento de Nutrição - UFPE.

Universidade Federal de Pernambuco em projeto colaborativo com a Prefeitura Municipal de Camaragibe, Pernambuco.

Correspondência: Diana Lemos Pessoa

Rua Gaspar Peres $n^{\circ} 427$, apt ${ }^{\circ} 204$ - Iputinga

50670-350 - Recife-PE - Brasil

Tel.: (81) 3453-3336

e-mail: diana.prof@yahoo.com.br 
hematológicos: hemoglobina $(\mathrm{Hb})$, hematócrito (Hct) e hemácias (He) e os indicadores hematimétricos: volume corpuscular médio (VCM), hemoglobina corpuscular média (HCM), concentração de hemoglobina corpuscular média (CHCM) e amplitude de distribuição dos eritrócitos (RDW). ${ }^{5}$

A concentração de hemoglobina é, atualmente, o parâmetro mais utilizado como indicativo das conseqüências fisiopatológicas da anemia. ${ }^{1}$ No entanto, a concentração de $\mathrm{Hb}$ não possui boa especificidade e sensibilidade para avaliar o estado nutricional do ferro, uma vez que pode se encontrar alterada em condições patológicas diversas, a exemplo dos processos infecciosos e inflamatórios, hemorragia, desnutrição protéico-calórica, uso de medicamentos e tabagismo. ${ }^{6}$ Por outro lado, ainda permanece controverso se concentrações de hemoglobina, em patamares mais baixos, representam apenas um fenômeno de ordem fisiológica ou o efetivo desenvolvimento de um substancial aumento na prevalência de anemia na população idosa. O VCM é um importante índice hematimétrico na medida em que sua determinação orienta o diagnóstico das anemias, classificando-as em microcíticas, normocíticas e macrocíticas. ${ }^{7}$ No entanto, o valor do VCM deve ser utilizado em conjunto com o do RDW. Nessa composição, o VCM indicaria a média do tamanho dos eritrócitos (normocíticos, macrocíticos ou microcíticos) e o RDW nortearia a interpretação da população de células eritróides, concernente à homogeineidade ou não da distribuição morfológica da massa eritrocitária. A combinação desses dois índices subsidiou uma nova classificação das anemias (Quadro 1).

Quadro 1

Classificação das anemias, segundo o VCM e o RDW.

\begin{tabular}{|c|c|c|}
\hline \multirow{2}{*}{ VCM (fL) } & \multicolumn{2}{|c|}{ RDW(\%) } \\
\hline & $\leq 14$ & $>14$ \\
\hline$<80$ & $\begin{array}{c}\text { Talassemia menor } \\
\text { Anemia por doença } \\
\text { crônica }\end{array}$ & Anemia ferropriva \\
\hline 80 |-|-| & $\begin{array}{l}\text { Anemia por } \\
\text { doença crônica } \\
\text { Hipotiroidismo }\end{array}$ & $\begin{array}{l}\text { Anemia sideroblástica } \\
\text { Síndrome mielodisplásica }\end{array}$ \\
\hline$>97$ & $\begin{array}{l}\text { Alcoolismo } \\
\text { Hepatopatias }\end{array}$ & $\begin{array}{c}\text { Anemia megaloblástica } \\
\text { Hemólise }\end{array}$ \\
\hline
\end{tabular}

Fonte: Failace, $1995^{8}$

\section{Casuística e métodos}

Desenho e população do estudo

O delineamento do estudo foi do tipo transversal, realizado em novembro/dezembro/2003. A população estudada compreendeu indivíduos de ambos os sexos com idade $\geq 60$ anos, no ano de 2003.

Critérios de exclusão

Foram excluídos do estudo indivíduos que referiram a ingestão de sais de ferro nos últimos trinta dias prévios à coleta de dados; indivíduos que, embora cadastrados no PSF, não se encontravam na base territorial englobada na seleção da amostra; ou que se recusaram a assinar o termo de consentimento para participação no estudo.

\section{Amostragem}

Para determinação do tamanho amostral, foi desenvolvido um estudo piloto que forneceu a estimativa da prevalência de anemia ( $\mathrm{p}=11,0 \%)$, na população de estudo ( $\mathrm{N}=$ 2.500). Adotando-se uma precisão de $3,5 \%$ (d) e uma confiabilidade de $95 \%$ ( $\mathrm{z}=1,96)$, o tamanho amostral mínimo, calculado a partir da fórmula $\mathrm{n}=[$ z.p.(1-p)]/d², ajustada para uma população finita, ${ }^{9}$ pela fórmula $n_{1}=[\mathrm{n} /[1+(\mathrm{n} / \mathrm{N})]$, foi de 273 indivíduos. Objetivando corrigir as eventuais perdas foi acrescido um percentual de cerca de 7\%, totalizando 292 idosos utilizados no processo de seleção. A casualização das unidades amostrais foi realizada utilizando-se um processo do tipo aleatório, segundo a técnica de amostragem sistemática, fazendo-se uso de uma tabela de números aleatórios.

\section{Exames laboratoriais}

A colheita matinal (8:00-10:00h) constituiu-se na obtenção de uma alíquota de 5,0 ml de sangue, mediante venipuntura braquial, e encaminhada ao Laboratório de Análises Clínicas para realização das análises hematológicas e hematimétricas, em contador eletrônico de células (Sismex SF 3000 Automated Hematology Analyser, GMI, Inc. Ramsey, MN, USA). Foram analisados os parâmetros descritos no Quadro 2, com os respectivos valores de referência.

Quadro 2

Valores de referência dos parâmetros hematológicos e hematimétricos, segundo o sexo

\begin{tabular}{ccc}
\hline Parâmetros & Mulheres & Homens \\
\hline $\mathrm{Hb}(\mathrm{g} / \mathrm{dL})$ & $<12,0$ & $<13,0$ \\
$\mathrm{VCM}(\mathrm{fL})$ & $81,0+-\mid 99,0$ & $80,0|-| 98,0$ \\
$\mathrm{CHCM}(\%)$ & $32,0+-\mid 35,8$ & $32,0|-| 37,0$ \\
$\mathrm{RDW}(\%)^{\mathrm{a}}$ & $10,0|-| 15,0$ & $10,0|-| 15,0$ \\
\hline
\end{tabular}

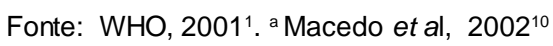

\section{Análise estatística}

Os dados foram digitados com dupla entrada e verificados com o Validate, módulo do Programa Epi-info, (Epi-info 6.04, WHO/CDC, Atlanta, GE, USA), para checar a consistência e validação. Os resultados foram descritos sob a forma de freqüências absolutas, relativas e acumuladas. Na descrição das proporções, a distribuição binomial foi aproximada à distribuição normal, pelo intervalo de confiança de 95\%. Nos testes de inferência estatística, as proporções foram comparadas pelo teste do Qui quadrado de Pearson. Foi utilizado o nível de significância de 5\% para rejeição de hipótese de 
nulidade. As análises estatísticas foram realizadas pelo programa SPSS for Windows, versão 13.1 (SPSS Inc, Chicago, IL, USA).

\section{Resultados}

População estudada e perdas

Foram estudados 284 idosos. As concentrações de $\mathrm{Hb}$ e os valores de CHCM foram avaliados em 284 idosos, o VCM foi mensurado em 282 e o RDW em 275. As perdas ocorridas foram relacionadas, sobretudo, à falta de consistência dos dados de algumas variáveis demográficas. No que diz respeito aos dados hematológicos e hematimétricos, as perdas se deveram, principalmente, à recusa à punção venosa, volume insuficiente de sangue para as análises, bem como aquelas inerentes ao próprio processo de análise laboratorial. No caso específico do RDW, foram excluídos da análise todos os histogramas que apresentavam distribuição bi ou multimodal.

\section{Prevalência de anemia}

A prevalência de anemia, segundo as concentrações de $\mathrm{Hb}$, foi de 10,9\% (IC 95\% 8,3\%-18,7\%), para o sexo masculino ( $\mathrm{Hb}<13 \mathrm{~g} / \mathrm{dl}$ ), e 12,6\% (IC 95\% 6,0\%-18,6\%) para o sexo feminino $(\mathrm{Hb}<12 \mathrm{~g} / \mathrm{dl})$, conforme demonstrado na tabela 1 .

\section{Morfologia eritrocitária}

A avaliação da morfologia eritrocitária, segundo os índices hematimétricos VCM e RDW, mostrou que 78,6\% (IC 95\% 58,5\% - 91,0\%) dos idosos anêmicos apresentaram concomitantemente isocitose e normocitose. No entanto, dos idosos anêmicos que apresentaram anisocitose (21,4\% (IC 95\% 9,0\%-41,5\%)), 10,7\% ( IC 95\% 2,8\%-29,4\%) apresentaram microcitose e 10,7\% ( IC 95\% 2,8\%-29,4\%) normocitose (Tabela 2).

Esses dados são sugestivos de que a etiologia da anemia, nesse grupo populacional, parece ser, na sua grande maioria, decorrente da doença crônica, enquanto as outras causas de anemia, incluindo a ferropênica, contribuíram com apenas $1 / 5$ dos casos totais de anemia.

Tabela 1

Distribuição das concentrações de hemoglobina em idosos do PSF segundo o sexo. Camaragibe, PE - 2003

\begin{tabular}{cccccccccc}
\hline$H b(g / d L)$ & $n$ & $\begin{array}{c}\text { Masculino } \\
\%\end{array}$ & $F A^{*}$ & $I C^{\star *}$ & $n$ & $\begin{array}{c}\text { Feminino } \\
\%\end{array}$ & $F A^{*}$ & $I C^{\star *}$ & $p^{\star \star *}$ \\
\hline$<11$ & 2 & 1,8 & 1,8 & $0,3-7,1$ & 4 & 2,3 & 2,3 & $0,7-6,2$ & 0,66 \\
$11+12$ & 3 & 2,7 & 4,5 & $1,7-10,8$ & 18 & 10,3 & 12,6 & $8,3-18,7$ & \\
12 - 13 & 7 & 6,4 & 10,9 & $8,3-18,7$ & 50 & 28,7 & 41,4 & $34,1-49,1$ & \\
$\geq 13$ & 98 & 89,1 & 100 & - & 102 & 58,6 & 100 & - & \\
Total & 110 & - & - & - & 174 & - & - & - & \\
\hline
\end{tabular}

Freqüência acumulada. ** Intervalo de confiança de 95\% *** Teste do qui-quadrado de Pearson

\section{Caracterização das anemias}

Concernente à caracterização das anemias, segundo a concentração de $\mathrm{Hb}$, a morfologia (VCM e RDW) e a coloração eritrocitária (CHCM), observou-se que apenas 8,0\% (IC 95\% 1,4\%-27,5\%) dos idosos anêmicos apresentaram anemia do tipo hipocrômica e microcítica, sugestiva de deficiência de ferro severa; 8,0\% (IC 95\% 1,4\%-27,5\%) anemia do tipo hipocrômica e normocítica e que apenas 4,0\% (IC 95\% 1,2\%-22,3\%) anemia microcítica isolada. De modo geral, a maior parte dos casos de anemia, diagnosticada pela concentração de hemoglobina e pelos valores de RDW, não apresentou alteração na morfologia e coloração eritrocitária, segundo VCM e CHCM, respectivamente (Tabela 3).

\section{Discussão}

A relativamente baixa prevalência de anemia, observada nos idosos de Camaragibe, quando utilizado o indicador $\mathrm{Hb}$, foi, de certa forma, similar àquelas prevalências encontradas em estudos com idosos residentes nas comunidades americanas. Nesse sentido, Inelman et al. ${ }^{11}$ relataram uma prevalência de 9,1\%, enquanto Skjelbakken et al. ${ }^{12}$ descreveram prevalência de $23,0 \%$, nesse mesmo grupo etário. Por sua vez, Timiras \& Brownstein ${ }^{13}$ reportaram, em idosos institucionalizados em clínicas geriátricas nos Estados Unidos, uma prevalência de 13,0\% de anemia. No entanto, tanto na Europa quanto nos Estados Unidos, têm sido descritas elevadas prevalências na população idosa hospitalizada, em patamares que ultrapassam a cifra de $30,0 \%{ }^{14}$ e $50 \%{ }^{15,16}$ Essa diferença de magnitude em idosos hospitalizados tem sido atribuída, sobretudo, à presença de doenças inflamatórias crônicas. A similaridade da prevalência estimada, em nossa casuística, com as prevalências encontradas em idosos americanos e europeus, inseridos em contexto socioeconômico diferentes do brasileiro, poderia ser atribuída a alguns fatores que merecem consideração. É plausível que a população estudada em Camaragibe, em virtude de estar fidelizada a um programa de prevenção e atenção à saúde, pode direta ou indiretamente estar protegida de condições adversas que determinam a ocorrência de carências nutricionais específicas e/ou de comprometimento do estado nutricional. Seria igualmente plausível pensar que, embora a amostra estudada tenha sido de idosos que declararam não fazer uso de suplementos vitamínicos e minerais num período de trinta dias anterior à coleta de dados, parte significativa desses indivíduos fizessem uso desses suplementos e cuja informação não tenha sido detectada nos critérios de elegibilidade, por conseguinte não excluídos do estudo. Seria perti- 
Tabela 2

Classificação da morfologia eritrocitária† dos casos de anemia segundo o RDW e o VCM de idosos do PSF. Camaragibe, PE-2003.

\begin{tabular}{|c|c|c|c|c|c|c|c|}
\hline \multirow[t]{3}{*}{ VCM } & \multicolumn{7}{|c|}{ RDW } \\
\hline & \multicolumn{3}{|c|}{ Isocitose+ } & \multicolumn{3}{|c|}{ Anisocitose++ } & \multirow[t]{2}{*}{ Total } \\
\hline & $\mathrm{n}$ & $\%$ & $\mathrm{IC}^{\dagger+}$ & $\mathrm{n}$ & $\%$ & $\mathrm{IC}^{+\dagger}$ & \\
\hline Microcitose* & 0,0 & 0,0 & - & 3,0 & 10,7 & $2,8-29,4$ & 3,0 \\
\hline Normocitose ${ }^{\star *}$ & 22,0 & 78,6 & $88,5-91,0$ & 3,0 & 10,7 & $2,8-29,4$ & 25,0 \\
\hline Macrocitose ${ }^{\star \star \star}$ & 0,0 & 0,0 & - & 0,0 & 0,0 & - & 0,0 \\
\hline Total & 22,0 & 78,6 & $88,5-91,0$ & 6,0 & 21,4 & $9,0-41,5$ & 28,0 \\
\hline
\end{tabular}

${ }^{\dagger}$ Adaptação de Failace, $1995^{8}$ e Wintrobe $1998^{7} ; *$ VCM $>81 \mathrm{fL}$ sexo fem.; VCM $>80 \mathrm{fL}$ sexo masc. ${ }^{* \star}$ VCM $81 \mathrm{fL} \mathrm{|-} \mathrm{-|} 99 \mathrm{fL}$ sexo fem.; VCM $80 \mathrm{fL}|--| 98$ fL sexo masc.;

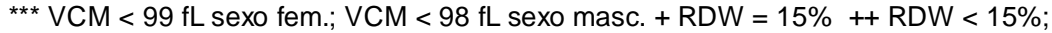
${ }^{\dagger \dagger}$ Intervalo de confiança de $95 \%$

Tabela 3

Classificação da anemia, segundo a concentração de $\mathrm{Hb}$, a morfologia eritrocitária (VCM, RDW) e a coloração eritrocitária ( $\mathrm{CHCM}$ ) de idosos do PSF. Camaragibe, PE-2003.

\begin{tabular}{|c|c|c|c|c|c|c|c|}
\hline \multirow{3}{*}{$\mathrm{CHCM}$} & \multirow{3}{*}{$\begin{array}{c}\text { VCM } \\
+ \\
\text { RDW }\end{array}$} & \multicolumn{6}{|c|}{$\mathrm{Hb}$} \\
\hline & & \multicolumn{2}{|c|}{ Anêmicos+ } & \multirow[b]{2}{*}{$\mathrm{IC}^{+\dagger}$} & \multicolumn{3}{|c|}{ Normais++ } \\
\hline & & $\mathrm{n}$ & $\%$ & & $\mathrm{n}$ & $\%$ & $\mathrm{IC}^{+\dagger}$ \\
\hline \multirow{4}{*}{ Hipocrômica* } & Microcítica• & 2,0 & 8,0 & $1,4-27,5$ & 1,0 & 0,5 & $0,1-2,9$ \\
\hline & Normocítica•• & 2,0 & 8,0 & $1,4-27,5$ & 15,0 & 6,9 & $4,0-11,3$ \\
\hline & Macrocítica••• & 0,0 & 0,0 & - & 0,0 & 0,0 & - \\
\hline & Microcítica & 1,0 & 4,0 & $0,2-22,3$ & 1,0 & 0,5 & $0,1-2,9$ \\
\hline \multirow[t]{2}{*}{ Normocrômica** } & Normocítica & 20,0 & 80,0 & $58,7-92,4$ & 198 & 90,8 & $86,0-94,2$ \\
\hline & Macrocítica & 0,0 & 0,0 & - & 3,0 & 1,4 & $0,4-4,3$ \\
\hline Total & & 25 & - & - & 218 & - & - \\
\hline
\end{tabular}

* $\mathrm{CHCM}>32 \%$ sexo masc. e fem.; ** 32\% - -| 35,8\% sexo fem.; $32 \%+-\mid 37,0 \%$ sexo masc.. $・$ VCM $>81 \mathrm{fL}$, RDW $<15 \%$ sexo fem.; VCM $>80 \mathrm{fL}$, RDW $<15 \%$ sexo masc. •. VCM 81fL - - $99 \mathrm{fL}$, RDW > 15\% sexo fem.; VCM $80 \mathrm{fL}$ - - $98 \mathrm{fL}$ RDW > 15\% sexo masc. $\cdots$ VCM $>99$ fLI RDW $<15 \%$ sexo fem.; $\mathrm{VCM}>98 \mathrm{fL}$, RDW $<15 \%$ sexo masc. $+\mathrm{Hb} \geq 12 \mathrm{~g} / \mathrm{dL}$ sexo fem.; $\mathrm{Hb} \geq 13 \mathrm{~g} / \mathrm{dL}$ sexo mas.; ++ $\mathrm{Hb}<12 \mathrm{~g} / \mathrm{dL}$ sexo fem.; $\mathrm{Hb}<13 \mathrm{~g} / \mathrm{dl}$ sexo masc.; ${ }^{\dagger \dagger}$ intervalo de confiança de 95\%

nente considerar ainda que parte desses idosos, durante o acompanhamento médico periódico, que constitui parte integrante no programa de atenção à saúde no qual estavam inscritos, já teria feito tratamento preventivo ou curativo com suplementação mineral e/ou vitamínica alguns meses anterior à coleta de dados e, portanto, temporariamente controlados da carência nutricional. Seria ainda importante ressaltar que a abordagem integral em saúde, prestada a essa clientela, deve incluir o combate às parasitoses intestinais, bem como orientação nutricional, que têm sido consideradas como medidas eficazes e efetivas no controle e prevenção da anemia.

A ausência de associação significativa entre a prevalência de anemia, segundo as concentrações de hemoglobina e o gênero, foi um resultado inesperado, considerando que o sexo masculino tem sido descrito como o de maior vulnerabilidade à ferropenia. Joosten et al ${ }^{16}$ e Timiras \& Brownstein ${ }^{13}$ encontraram prevalências maiores nos homens, chegando a uma diferença de até 22,0\% e 9,3\%, respectivamente, quando comparados àquelas observadas no sexo feminino. No entanto, não se tem conhecimento, até o presente, das razões dessa maior fragilidade dos indivíduos do sexo masculino à carência marcial. Têm sido levantadas como possíveis explicações para tal ocorrência as causas de ordem metodológica, traduzidas pelo uso de pontos de corte mais baixos para o sexo feminino. Na defesa dessa tese estaria a concepção de que os estrógenos, ao funcionarem como potenciais agentes inibidores da eritropoese, levariam o sexo feminino a ser mais vulnerável à anemia. Logo, os pontos de corte para o diagnóstico da anemia são mais baixos para o sexo feminino quando comparados com aqueles usados para o sexo masculino. No entanto, após a menopausa a redução dos níveis de estrógenos induziria a um aumento da massa eritrocitária a níveis similares ao observado em indivíduos do sexo masculino. Dessa forma, não se justificaria o uso de pontos de corte diferenciados para cada sexo., ${ }^{8,17}$

A ocorrência de apenas 21,4\% de microcitose ou normocitose associada à anisocitose, quando utilizados os índices hematimétricos VCM e RDW, é um forte indício de que a anemia presente, na maioria dos idosos, não seja decorrente de um estado ferropênico ou de outras anemias que não aquelas atribuídas à doença crônica.

A classificação de pacientes anêmicos, que apresentaram anisocitose associada à normocitose como ferropênicos, se deve ao fato de que a carência incipiente de ferro, com aporte à eritropoese que varie entre momentos de falta e de suficiência do mineral, gera uma coorte de eritrócitos com conteúdo hemoglobínico variado e conseqüente variação volumétrica. Dessa forma, o RDW aumenta antes de haver variação significativa do VCM. $^{8}$

$\mathrm{O}$ uso do $\mathrm{CHCM}$ como indicador de severidade da deficiência de ferro demonstrou que, dos casos de anemia ferropênica, a maioria apresentou alteração de CHCM, sugestiva de um quadro de carência severa, uma vez que alterações na coloração eritrocitária só aparecem quando concentrações de $\mathrm{Hb}$ apresentam cifras inferiores a $8 \mathrm{~g} / \mathrm{dl} .^{8}$

A reduzida associação entre os casos sugestivos de ferropenia com as concentrações inadequadas de hemo- 
globina foi confirmada pelos resultados dos índices hematimétricos VCM e RDW, onde cifras inferiores a 2,0\% desse tipo de anemia foram alcançadas em nossa casuística. Esses achados estão distanciados daqueles resultados que têm sido reportados em outros estudos. ${ }^{2,18}$ Para Failace, ${ }^{8}$ a avassaladora maioria dos casos de anemia por deficiência hemoglobínica é oriunda da deficiência de ferro.

A grande maioria dos casos classificados como anêmicos não apresentou alteração do volume corpuscular e da coloração eritrocitária, caracterizando um quadro de anemia por doença crônica, que parece ser o tipo de anemia dominante nesse grupo populacional. Esses resultados são coerentes com aqueles esperados, considerando que indivíduos idosos apresentam maior vulnerabilidade aos processos inflamatórios crônicos.

A prevalência de anemia em idosos fidelizados ao Programa de Saúde da Família de Camaragibe foi relativamente baixa quando comparada à magnitude de prevalências observadas em outros grupos etários do ciclo vital, sendo no entanto muito similar à ocorrência de anemia relatada em idosos de outros contextos ecológicos. A grande maioria dos idosos apresentou anemia do tipo normocrômica, normocítica e sem anisocitose, o que é sugestivo de anemia por doença crônica. Logo, a ínfima prevalência de anemia do tipo microcítica ou macrocítica, com anisocitose, minimiza a gênese da deficiência de ferro, vitamina $B_{12}$ ou ácido fólico na etiologia da anemia em idosos de Camaragibe.

\begin{abstract}
Anemia is a serious public health problem worldwide that mainly affects children and women of childbearing age. However, data about anemia in elderly individuals are still scarce in developing countries. This study aimed at assessing the prevalence and characteristics of anemia among an elderly population attending the Health Family Program in Camaragibe, northeast Brazil. Following a systematic sampling procedure, a cross-sectional study was carried out in November and December 2003 involving 284 subjects of both genders with ages grater than or equal to 60 years old. Anemia was estimated by the measurement of hemoglobin, mean cell hemoglobin concentration, mean cell volume and red cell distribution width. The prevalence of anemia was around $11.0 \%$. Red cell distribution width and mean cell volume were not correlated to hemoglobin count. The major characteristics of anemia were normochromia with normocytosis without anisocytosis suggesting anemia due to chronic diseases. The very low prevalence of microcytosis, macrocytosis and anisocytosis seems to be more likely due to causes other than iron, vitamin $B_{12}$ or folic acid deficiencies. The use of indicators that reflect the degree of anisocytosis, as well as parameters to assess iron status should be strongly recommended in the screening of anemia in the elderly population. Rev. bras. hematol. hemoter. 2006;28(4):288-292.
\end{abstract}

Key words: Anaemia; elderly; hematimetric indices; diagnosis; Health Family Programme.

\section{Agradecimentos}

Os autores agradecem à Fundação de Apoio à Pesquisa do Estado de Pernambuco (Facepe) e ao Conselho Nacional de Pesquisa em Ciência e Tecnologia-CNPQ, bem como à Prefeitura Municipal de Camaragibe, pelo suporte financeiro e logístico.

\section{Referências Bibliográficas}

1. World Health Organization. Iron deficiency anaemia. Assessment, prevention and control. A guide for programme managers. Geneva: WHO 2001;114p.

2. Beghé C, Wilson A, Ershler WB. Prevalence and outcomes of anemia in geriatrics: a systematic review of the literature. Am J Med 2004;116:3-10.

3. Choi CW, Cho WR, Park KH, et al. The cutoff value of serum ferritin for the diagnosis of iron deficiency in community-residing older persons. Ann Hematol 2005;84:358-91.

4. Jack M, Guralnick RS, Eisenstaedt LF, et al. Prevalence of anemia in persons 65 years and older in the Unites States: evidence for a high rate of unexplained anemia. Blood 2004;104:2263-68.

5. Mukhopadhyay D, Mohanaruban K. Iron deficiency anaemia in older people: investigation, management and treatment. Age and Ageing, Br Geriatr Soc 2002;31:87-91.

6. Paiva AA, Rondó HCP, Shinohara EMG. Parâmetros de avaliação do estado nutricional do ferro. Rev Saúde Pública 2000;34:1-11.

7. Wintrobe, MM. Hematologia Clínica. São Paulo, $1^{\text {a }}$ ed, 2v, Manole, 1998; v.2: 825-1100.

8. Failace R. Hemograma: Manual de interpretação. $3^{\mathrm{a}}$ ed. Porto Alegre: Artmed, 1995. 197p.

9. Bland M. An Introduction to Medical Statistics. $3^{\text {rd }}$ Ed. Oxford: Oxford University Press, 2000. 405p.

10. Macedo LA. Distúrbios hematológicos em indivíduos idosos. In: Freitas EV, Rocha SM, et al. Tratado de Geriatria e Gerontologia. Rio de Janeiro: Guanabara, 2002. p. 699 -707.

11. Inelman EM, D`Alessio M, Gatto MR, et al. Descriptive analysis of the prevalence of anemia in a randomly selected sample of elderly people living at home: some results of an Italian multicentric study. Aging 1994;6:81-9.

12. Skjelbakken T, Langbakk B, Lochen ML, et al. Haemoglobin and anaemia in a gender perspective: The Tromso Study. Eur J Hematol. 2005;74:381-88.

13. Timiras MT, Brownstein H. Prevalence of anemia and correlation of hemoglobin with age in a geriatric screening clinic population. J Am Geriatr Soc 1987;37:639-43.

14. Lipschitz DA, Mitchel CO, Thompson C. The anemia of senescence. Am J Hematol 1981;11:47-54.

15. Bird T, Hall MR, Sehade RO. Gastric histology and its relation to anaemia in the elderly. Gerontology 1977;23:309-21.

16. Joosten E, Pelemans W, Heile M et al. Prevalence and causes of anaemia in a geriatric hospitalized population. Gerontology 1992; $38: 1-2$.

17. Sahadevan S, Choo PW, Jayaratnam F.J. Anaemia in the hospitalised elderly. Singapore Med J 1995;35:375-78.

18. Daly MP. Anaemia in the elderly. Am Fam Physician 2000;62:751565.

Avaliação: Editor e dois revisores externos

Conflito de interesse: não declarado

Recebido: 24/06/2006

Aceito: 21/08/2006 